\title{
Reanalysis techniques in stochastic analysis of linear structures under stationary multi-correlated input
}

\author{
by \\ P. Cacciola ${ }^{1}$ and G. Muscolino ${ }^{2}$ \\ ${ }^{1}$ School of Environment and Technology, University of Brighton, Lewes road, BN24GJ, Brighton, UK \\ ${ }^{2}$ D.I.C- Dipartimento di Ingegneria Civile - C.I.Di.S.-Centro Interuniversitario di Dinamica Strutturale \\ Teorica e Sperimentale, Università di Messina, Vill. S.Agata, 98166, Messina , Italy
}

\begin{abstract}
The aim of the Reanalysis is determining the structural response of modified systems using the pertinent results from the original or "reference" structure, so reducing the computational effort. Repeated analyses of structures under certain or uncertain loads are often necessary in various fields of applications. Optimization techniques, model updating, design process, Monte Carlo simulations of structures with uncertain parameters are some examples in which several analyses of slightly modified systems occurs. In order to reduce the computational effort in determining both the static and the dynamic response, various Reanalysis techniques have been proposed in literature. In this paper the main static Reanalysis techniques are reformulated to perform the Reanalysis of linear structural systems subjected to multi-correlated stationary Gaussian stochastic input for both topological and nontopological structural modifications.
\end{abstract}

Keywords: Reanalysis, dynamic response, stationary random response.

\section{Introduction}

Repeated analysis, or "Reanalysis", is needed in various fields of structural applications, as design, structural optimization, model updating and structural damage analysis. In these cases, indeed, various modifications that require repetitive analysis of modified systems can occur. The aim of the Reanalysis is the evaluation of the structural response (e.g. displacements, forces and stresses) for such changes without solving the complete set of modified algebraic and/or differential equations. The solution procedures usually use the results from the analysis of the original structure, called as "reference" structure, so reducing the computational effort. The interest in the Reanalysis techniques are increased drastically in last two decades, in spite of the significant increase in computer processing power, memory and storage space. 
During design, optimization, model updating and damage analysis processes, mechanical and geometrical parameters may change and eventually structural components can be added or deleted leading also to a change of the number of the degrees of freedom of the the pertinent finite element model. It follows that the Reanalysis techniques are classified as "topological" or "non-topological" if the modifications lead to a change of the degrees of freedom of the system or not. Moreover, the Reanalysis techniques can be used to evaluate the response of structures with linear and non-linear behaviour (see e.g. Kirsch 2008).

In the framework of static Reanalysis, recently Akgun et al. (2001) described and compared three methods - the combined approximations, theorems of structural variations and virtual distorsion method; they show that all the methods stemming from static Reanalysis are equivalent (for truss structures) to the Sherman-Morrison $(1949,1950)$ and Woodbury (1950) formulas. A review of the main contributions in Vibration Reanalysis can be found in Kirsch (2008). In this item the main computational effort is spent in the solution of the eigenproblem, because of exact solution techniques of the problem can be prohibitively expensive or unattainable. The deterministic evaluation of dynamic response in the time domain, usually called Dynamic Reanalysis, has been performed through the extension to the dynamics of the Combined Approximations approach (see e.g. Kirsch 2008), the virtual distorsion method (see Kolakowski et al 2008) or by applying the so-called dynamics modification method to the Reanalysis (Muscolino and Cacciola 2004, Cacciola et al 2005). All the previously quoted methods are able to evaluate dynamic responses of either linear or non-linear systems. In the combined approximation approach (Kirsch 1996, 2008) global expressions can be achieved by considering as basis vectors terms of local approximations. In the virtual distorsion method, virtual forces, modelling modifications of mass distribution, as well as virtual distortions, modelling stiffness modifications of structural elements, are introduced (Kolakowski et al 2008) to modify the reference structure in a fast and efficient way. The basic idea of third method is grounded on the assumption that all the dynamic modifications can be viewed as pseudo-forces, according to the socalled dynamic modification method (Muscolino 1996). The response of the modified structure is retrieved starting from the knowledge of the transition matrix and the eigenproperties of the original structure. So that the main difference with the classical Vibration Reanalysis is that the eigenproblem Reanalysis is avoided drastically reducing the computational effort. Therefore, the transient and loading operators involved in the step-by-step numerical procedure are determined in appropriate form from the knowledge of the original ones related to the unmodified structure. Remarkably, it has been shown that the third approach is also computationally very effective in determining the random response of multi-degreeof-freedom (MDoF) systems with random parameters via a pertinent Monte Carlo simulation (Muscolino and Cacciola 2004). Moreover, via this approach the response of non-classically damped system is determined without the evaluation of complex quantities. The latter method has been extended to the Dynamic Reanalysis of linear systems subjected to stochastic excitation, modelled as a white noise processes (Muscolino and Cacciola 2004, Cacciola et al 2003, 2005). 
In this paper the Dynamic Reanalysis of linear structural systems, with both topological and non-topological modifications, under multi-correlated stationary Gaussian non-white excitations is performed. In particular the Kroneker algebra is extensively adopted in order to evaluate the first and second order statistical moments of the response, of both original and modified structures, as the solution of two sets of algebraic equations (Muscolino 1996). The procedure preliminarily requires the projection of the equations governing the evolution of the statistical moments in a reduced space, by means of a coordinate transformation which requires the evaluation of the eigenproperties of original structure only. To this aim the eigenproperties of the original system are suitably exploited so avoiding the evaluation of the eigenproperties of the modified system. Therefore, the main methods of static Reanalysis are reformulated in order to evaluate the statistics of the modified structural system, with reduced computational efforts.

The numerical results show the accuracy of the proposed approaches for the analysis of multi-degrees-of-freedom (MDoF) systems. Computational aspects are also addressed.

\section{Basic equations}

\subsection{Deterministic analysis}

Consider an $n$-DoF quiescent classically damped structure whose dynamic behaviour is governed by the following equations of motion

$$
\mathbf{M}_{\mathrm{o}} \ddot{\mathbf{u}}_{\mathrm{o}}(t)+\mathbf{C}_{\mathrm{o}} \dot{\mathbf{u}}_{\mathrm{o}}(t)+\mathbf{K}_{\mathrm{o}} \mathbf{u}_{\mathrm{o}}(t)=\mathbf{f}_{\mathrm{o}}(t) ; \quad \mathbf{u}_{\mathrm{o}}(0)=\mathbf{0} ; \dot{\mathbf{u}}_{\mathrm{o}}(0)=\mathbf{0},
$$

where $\mathbf{M}_{\mathrm{o}}, \mathbf{C}_{\mathrm{o}}$ and $\mathbf{K}_{\mathrm{o}}$ are the mass, damping and stiffness matrices of order $n \times n$, respectively; $\mathbf{u}_{\mathrm{o}}(t)$ is the $n \times 1$ vector listing the nodal displacements and $\mathbf{f}_{\mathrm{o}}(t)$ is the $n \times 1$ load vector of the nodal time-varying applied forces; $\mathbf{u}_{\mathrm{o}}(0)$ and $\dot{\mathbf{u}}_{\mathrm{o}}(0)$ are the initial conditions and the dot over a variable or a vector defines the time derivative. Note that the classically damped system has been chosen for sake of convenience. Indeed assuming the original system as classically damped structure, it is possible to uncouple the equations of motion by the following modal coordinate transformation

$$
\mathbf{u}_{\mathrm{o}}(t)=\Phi_{\mathrm{o}} \mathbf{q}_{\mathrm{o}}(t)
$$

where $\mathbf{q}_{\mathrm{o}}(t)$ is the $s \times 1(s \leq n)$ vector of the generalized coordinates and $\boldsymbol{\Phi}_{\mathrm{o}}$ is the modal matrix of order $n \times s$, solution of the following eigenproblem

$$
\mathbf{K}_{\mathrm{o}} \boldsymbol{\Phi}_{\mathrm{o}}=\mathbf{M}_{\mathrm{o}} \boldsymbol{\Phi}_{\mathrm{o}} \boldsymbol{\Omega}_{\mathrm{o}}{ }^{2}, \quad \boldsymbol{\Phi}_{\mathrm{o}}{ }^{T} \mathbf{M}_{\mathrm{o}} \boldsymbol{\Phi}_{\mathrm{o}}=\mathbf{I}_{s},
$$


in which $\Omega_{\mathrm{o}}$ is the diagonal matrix listing the first $s$ natural circular frequencies $\omega_{\mathrm{o}, j}$ of the original or "reference" structure and $\mathbf{I}_{s}$ is the identity matrix of order $s \times s$. The uncoupled equations of motion, in the reduced modal space, can be written as

$$
\ddot{\mathbf{q}}_{\mathrm{o}}(t)+\boldsymbol{\Xi}_{\mathrm{o}} \dot{\mathbf{q}}_{\mathrm{o}}(t)+\boldsymbol{\Omega}_{\mathrm{o}}^{2} \mathbf{q}_{\mathrm{o}}(t)=\boldsymbol{\Phi}_{\mathrm{o}}^{T} \mathbf{f}_{\mathrm{o}}(t) ; \quad \mathbf{q}_{\mathrm{o}}(0)=\mathbf{0}, \quad \dot{\mathbf{q}}_{\mathrm{o}}(0)=\mathbf{0},
$$

where $\Xi_{0}$ is the diagonal generalized damping matrix whose $j$-th elements is $2 \zeta_{\mathrm{o}, j} \omega_{\mathrm{o}, j}$, with $\zeta_{\mathrm{o}, j}$ the damping ratio of the $j$-th mode. In order to evaluate the response, Equation (4) can be conveniently rewritten introducing the $2 s \times 1$ vector of the reduced modal state variables $\mathbf{z}_{\mathrm{o}}(t)$

$$
\dot{\mathbf{z}}_{\mathrm{o}}(t)=\mathbf{D}_{\mathrm{o}} \mathbf{z}_{\mathrm{o}}(t)+\mathbf{V}_{\mathrm{o}} \mathbf{f}_{\mathrm{o}}(t) ; \quad \mathbf{z}_{\mathrm{o}}(0)=\mathbf{0},
$$

being

$$
\mathbf{z}_{\mathrm{o}}(t)=\left[\begin{array}{c}
\mathbf{q}_{\mathrm{o}}(t) \\
\dot{\mathbf{q}}_{\mathrm{o}}(t)
\end{array}\right] ; \quad \mathbf{D}_{\mathrm{o}}=\left[\begin{array}{cc}
\mathbf{0} & \mathbf{I}_{s} \\
-\mathbf{\Omega}_{\mathrm{o}}{ }^{2} & -\mathbf{\Xi}_{\mathrm{o}}
\end{array}\right] ; \quad \mathbf{V}_{\mathrm{o}}=\left[\begin{array}{c}
\mathbf{0} \\
\boldsymbol{\Phi}_{\mathrm{o}}{ }^{\mathrm{T}}
\end{array}\right]
$$

Once the modal response in the state variable has been evaluated, the nodal state vector response can be obtained by the following relationship

$$
\mathbf{y}_{\mathrm{o}}(t)=\Pi_{\mathrm{o}} \mathbf{z}_{\mathrm{o}}(t) ; \quad \mathbf{y}_{\mathrm{o}}(t)=\left[\begin{array}{c}
\mathbf{u}_{\mathrm{o}}(t) \\
\dot{\mathbf{u}}_{\mathrm{o}}(t)
\end{array}\right]
$$

where the matrix $\Pi_{\mathrm{o}}$ of order $2 n \times 2 s$ is given by

$$
\Pi_{\mathrm{o}}=\left[\begin{array}{cc}
\Phi_{\mathrm{o}} & \mathbf{0} \\
\mathbf{0} & \boldsymbol{\Phi}_{\mathrm{o}}
\end{array}\right]
$$

\subsection{Stochastic analysis}

Let us consider a linear system subjected to stochastic processes. The external load vector $\mathbf{f}_{\mathrm{o}}(t)$ can be modelled as a multi-correlated Gaussian stochastic process fully characterized by the mean vector, $\boldsymbol{\mu}_{\mathbf{f}_{\mathrm{o}}}(t)$, and correlation vector, $\mathbf{r}_{\mathbf{f}_{0} \mathbf{f}_{\mathrm{o}}}\left(t_{1}, t_{2}\right)$, given by

$$
\begin{aligned}
& \boldsymbol{\mu}_{\mathbf{f}_{\mathrm{o}}}(t)=\mathrm{E}\left\langle\mathbf{f}_{\mathrm{o}}(t)\right\rangle ; \\
& \mathbf{r}_{\mathbf{f}_{\mathrm{o}} \mathbf{f}_{\mathrm{o}}}\left(t_{1}, t_{2}\right)=\mathrm{E}\left\langle\mathbf{f}_{\mathrm{o}}\left(t_{1}\right) \otimes \mathbf{f}_{\mathrm{o}}\left(t_{2}\right)\right\rangle-\boldsymbol{\mu}_{\mathbf{f}_{\mathrm{o}}}\left(t_{1}\right) \otimes \boldsymbol{\mu}_{\mathbf{f}_{\mathrm{o}}}\left(t_{2}\right),
\end{aligned}
$$


where $\mathrm{E}\langle\cdot\rangle$ denotes the stochastic average and the symbol $\otimes$ means Kronecker product (Brewer 1978). The input process $\mathbf{f}_{\mathrm{o}}(t)$ can be conveniently split in two terms as follows

$$
\mathbf{f}_{\mathrm{o}}(t)=\mu_{\mathbf{f}_{\mathrm{o}}}(t)+\tilde{\mathbf{X}}_{\mathbf{f}_{\mathrm{o}}}(t)
$$

In this equation $\mu_{\mathbf{f}_{\mathrm{o}}}(t)$ is a deterministic vector, whereas $\tilde{\mathbf{X}}_{\mathbf{f}_{\mathrm{o}}}(t)$ is a zero-mean multi-correlated Gaussian random process, with correlation vector $\mathbf{r}_{\tilde{\mathbf{X}}_{\mathbf{f}_{\mathrm{o}}} \tilde{\mathbf{x}}_{\mathbf{f}_{\mathrm{o}}}}\left(t_{1}, t_{2}\right)=\mathrm{E}\left\langle\tilde{\mathbf{X}}_{\mathbf{f}_{\mathrm{o}}}\left(t_{1}\right) \otimes \tilde{\mathbf{X}}_{\mathbf{f}_{\mathrm{o}}}\left(t_{2}\right)\right\rangle \equiv \mathbf{r}_{\mathbf{f}_{\mathrm{o}} \mathbf{f}_{\mathrm{o}}}\left(t_{1}, t_{2}\right)$, accounting for the contribution due to the stochastic excitation. In view of the linearity of the problem under consideration and taking into account Equation (10) the state vector $\mathbf{z}_{\mathrm{o}}(t)$ in modal space can expressed as follows

$$
\mathbf{z}_{\mathrm{o}}(t)=\mu_{\mathbf{z}_{\mathrm{o}}}(t)+\tilde{\mathbf{Z}}_{\mathrm{o}}(t)
$$

where the first term $\boldsymbol{\mu}_{\mathbf{z}_{\mathrm{o}}}(t)=\mathrm{E}\left\langle\mathbf{z}_{\mathrm{o}}(t)\right\rangle$ represents the deterministic response associated with the mean value load vector $\mu_{\mathbf{f}_{\mathrm{o}}}(t)$, whereas $\tilde{\mathbf{Z}}_{\mathrm{o}}(t)$ is a zero mean $2 s$-variate random process, solution of the following differential equations

$$
\dot{\tilde{\mathbf{Z}}}_{\mathrm{o}}(t)=\mathbf{D}_{\mathrm{o}} \tilde{\mathbf{Z}}_{\mathrm{o}}(t)+\mathbf{V}_{\mathrm{o}} \tilde{\mathbf{X}}_{\mathbf{f}_{\mathrm{o}}}(t) ; \quad \tilde{\mathbf{Z}}_{\mathrm{o}}(0)=\mathbf{0}
$$

In many cases of practical interest, the response is adequately characterized by the knowledge of the mean value vector $\mu_{\mathbf{z}_{\mathrm{o}}}(t)$ and of the covariance vector $\sigma_{\mathbf{z}_{\mathrm{o}}}(t) \equiv$ $\mathrm{E}\left\langle\tilde{\mathbf{Z}}_{\mathrm{o}}(t) \otimes \tilde{\mathbf{Z}}_{\mathrm{o}}(t)\right\rangle$. The vector $\boldsymbol{\sigma}_{\mathbf{z}_{\mathrm{o}}}(t)$ represents the vectorialized form of the crosscovariance matrix, i.e. $\boldsymbol{\sigma}_{\mathbf{z}_{\mathrm{o}}}(t) \equiv \operatorname{Vec}\left\{\mathrm{E}\left\langle\tilde{\mathbf{Z}}_{\mathrm{o}}(t) \tilde{\mathbf{Z}}_{\mathrm{o}}^{\mathrm{T}}(t)\right\rangle^{\mathrm{T}}\right\}$, that is a column vector formed by all columns of the matrix in parentheses in such a way that the columns are written one below each other.

After some algebra (Muscolino 1996, 2001), the differential equations ruling the time-evolution of the vectors $\mu_{\mathbf{z}_{\mathrm{o}}}(t)$ and $\sigma_{\mathbf{z}_{\mathrm{o}}}(t)$ can be written, respectively, as

$$
\begin{aligned}
& \dot{\mu}_{\mathbf{z}_{0}}(t)=\mathbf{D}_{\mathrm{o}} \mu_{\mathbf{z}_{\mathrm{o}}}(t)+\mathbf{V}_{\mathrm{o}} \boldsymbol{\mu}_{\mathbf{f}_{\mathrm{o}}}(t) \\
& \dot{\boldsymbol{\sigma}}_{\mathbf{z}_{\mathrm{o}}}(t)=\mathbf{D}_{\mathrm{o}, 2} \boldsymbol{\sigma}_{\mathbf{z}_{\mathrm{o}}}(t)+\tilde{\mathbf{F}}_{\mathbf{z}_{\mathrm{o}}}(t),
\end{aligned}
$$

Note that by employing the Kronecker algebra the evolution of the second order moment is reduced to a set of first-order ordinary differential equations formally 
analogous to the one derived for the deterministic case. In writing Equation (13) the following positions have been made

$\mathbf{D}_{\mathrm{o}, 2}=\mathbf{D}_{\mathrm{o}} \otimes \mathbf{I}_{2 s}+\mathbf{I}_{2 s} \otimes \mathbf{D}_{\mathrm{o}}$

$\tilde{\mathbf{F}}_{\mathbf{Z}_{\mathrm{o}}}(t)=\left(\mathbf{V}_{\mathrm{o}} \otimes \mathbf{I}_{2 s}\right) \mathrm{E}\left\langle\tilde{\mathbf{X}}_{\mathbf{f}_{\mathrm{o}}}(t) \otimes \tilde{\mathbf{Z}}_{\mathrm{o}}(t)\right\rangle+\left(\mathbf{I}_{2 s} \otimes \mathbf{V}_{\mathrm{o}}\right) \mathrm{E}\left\langle\tilde{\mathbf{Z}}_{\mathrm{o}}(t) \otimes \tilde{\mathbf{X}}_{\mathbf{f}_{\mathrm{o}}}(t)\right\rangle$,

where $\mathbf{D}_{\mathrm{o}, 2}$ is a matrix of order $4 s^{2} \times 4 s^{2}$ and $\tilde{\mathbf{F}}_{\mathbf{Z}_{\mathrm{o}}}(t)$ is a deterministic vector of order $4 s^{2}$. After very simple algebra, the stochastic averages $\mathrm{E}\left\langle\tilde{\mathbf{X}}_{\mathbf{f}_{\mathrm{o}}}(t) \otimes \tilde{\mathbf{Z}}_{\mathrm{o}}(t)\right\rangle$ and $\mathrm{E}\left\langle\tilde{\mathbf{Z}}_{\mathrm{o}}(t) \otimes \tilde{\mathbf{X}}_{\mathbf{f}_{\mathrm{o}}}(t)\right\rangle$, which appear in the second Equation (14), can be evaluated, respectively, as follows

$$
\begin{aligned}
& \mathrm{E}\left\langle\tilde{\mathbf{X}}_{\mathbf{f}_{\mathrm{o}}}(t) \otimes \tilde{\mathbf{Z}}_{\mathrm{o}}(t)\right\rangle=\int_{0}^{t}\left[\mathbf{I}_{n} \otimes\left(\Theta_{\mathrm{o}}(t-\tau) \mathbf{V}_{\mathrm{o}}\right)\right] \mathbf{r}_{\tilde{\mathbf{X}}_{\mathbf{f}_{\mathrm{o}}} \tilde{\mathbf{X}}_{\mathbf{f}_{\mathrm{o}}}(t, \tau) \mathrm{d} \tau} \\
& \mathrm{E}\left\langle\tilde{\mathbf{Z}}_{\mathrm{o}}(t) \otimes \tilde{\mathbf{X}}_{\mathbf{f}_{\mathrm{o}}}(t)\right\rangle=\int_{0}^{t}\left[\left(\Theta_{\mathrm{o}}(t-\tau) \mathbf{V}_{\mathrm{o}}\right) \otimes \mathbf{I}_{n}\right] \mathbf{r}_{\tilde{\mathbf{X}}_{\mathbf{f}_{\mathrm{o}}} \tilde{\mathbf{X}}_{\mathbf{f}_{\mathrm{o}}}}(\tau, t) \mathrm{d} \tau
\end{aligned}
$$

For stationary excitations the response is governed by the solution of the algebraic equations obtained removing the time dependence from Equation (13) obtaining (Muscolino 1996, 2001):

$\mathbf{D}_{\mathrm{o}} \boldsymbol{\mu}_{\mathrm{Z}_{\mathrm{o}}}+\mathbf{V}_{\mathrm{o}} \boldsymbol{\mu}_{\mathrm{f}_{\mathrm{o}}}=0$

$\mathbf{D}_{\mathrm{o}, 2} \sigma_{\mathbf{Z}_{\mathrm{o}}}+\tilde{\mathbf{F}}_{\mathbf{Z}_{\mathrm{o}}}=\mathbf{0}$

where the elements of vector $\tilde{\mathbf{F}}_{\mathbf{Z}_{\mathrm{o}}}$ can be evaluated as:

$$
\begin{aligned}
& \mathrm{E}\left\langle\tilde{\mathbf{X}}_{\mathbf{f}_{\mathrm{o}}}(t) \otimes \tilde{\mathbf{Z}}_{\mathrm{o}}(t)\right\rangle=\int_{0}^{\infty}\left[\mathbf{I}_{n} \otimes\left(\Theta_{\mathrm{o}}(t-\tau) \mathbf{V}_{\mathrm{o}}\right)\right] \mathbf{r}_{\tilde{\mathbf{X}}_{\mathbf{f}_{\mathrm{o}}} \tilde{\mathbf{X}}_{\mathbf{f}_{\mathrm{o}}}(\tau-t) \mathrm{d} \tau ;} \\
& \mathrm{E}\left\langle\tilde{\mathbf{Z}}_{\mathrm{o}}(t) \otimes \tilde{\mathbf{X}}_{\mathbf{f}_{\mathrm{o}}}(t)\right\rangle=\int_{0}^{\infty}\left[\left(\Theta_{\mathrm{o}}(t-\tau) \mathbf{V}_{\mathrm{o}}\right) \otimes \mathbf{I}_{n}\right] \mathbf{r}_{\tilde{\mathbf{X}}_{\mathbf{f}_{\mathrm{o}}} \tilde{\mathbf{X}}_{\mathbf{f}_{\mathrm{o}}}}(t-\tau) \mathrm{d} \tau .
\end{aligned}
$$

In the frequency domain the stationary stochastic zero-mean process is fully defined by the power spectral density (PSD) function vector $\mathbf{s}_{\tilde{\mathbf{x}}_{\mathbf{f}_{0}} \tilde{\mathbf{x}}_{\mathbf{f}_{0}}}(\omega)$. The two function vectors are related by the following relationships: 
$\mathbf{r}_{\tilde{\mathbf{X}}_{\mathbf{f}_{0}} \tilde{\mathbf{X}}_{\mathbf{f}_{0}}}(t-\tau) \equiv \operatorname{Vec}\left\{\mathbf{R}_{\tilde{\mathbf{x}}_{\mathbf{f}_{0}}}^{\mathrm{T}} \tilde{\mathbf{x}}_{\mathbf{f}_{0}}(t-\tau)\right\}=\int_{-\infty}^{\infty} \exp [\mathrm{i} \omega(t-\tau)] \mathbf{s}_{\tilde{\mathbf{x}}_{\mathbf{f}_{0}} \tilde{\mathbf{x}}_{\mathrm{f}_{o}}}(\omega) \mathrm{d} \omega ;$

$\mathbf{r}_{\tilde{\mathbf{X}}_{\mathbf{f}_{0}} \tilde{\mathbf{X}}_{\mathbf{f}_{0}}}(\tau-t) \equiv \operatorname{Vec}\left\{\mathbf{R}_{\tilde{\mathbf{X}}_{\mathrm{f}_{0}} \tilde{\mathbf{X}}_{\mathrm{f}_{0}}}^{\mathrm{T}}(\tau-t)\right\}=\int_{-\infty}^{\infty} \exp [\mathrm{i} \omega(\tau-t)] \mathbf{s}_{\tilde{\mathbf{X}}_{\mathrm{f}_{0}} \tilde{\mathbf{x}}_{\mathrm{f}_{0}}}(\omega) \mathrm{d} \omega$,

where $i=\sqrt{-1}$ is the imaginary unit and

$$
\mathbf{S}_{\tilde{\mathbf{X}}_{\mathbf{f}_{0}} \tilde{\mathbf{x}}_{\mathbf{f}_{\mathrm{o}}}}(\omega)=\operatorname{Vec}\left\{\mathbf{S}_{\tilde{\mathbf{X}}_{\mathbf{f}_{0}} \tilde{\mathbf{x}}_{\mathbf{f}_{0}}}^{\mathrm{T}}(\omega)\right\} \equiv \operatorname{Vec}\left\{\mathbf{S}_{\tilde{\mathbf{X}}_{\mathrm{f}_{0}} \tilde{\mathbf{x}}_{\mathbf{f}_{0}}}(\omega)\right\}
$$

In this equation $\mathbf{S}_{\tilde{\mathbf{X}}_{\mathrm{f}} \tilde{\mathbf{X}}_{\mathrm{f}}}(\omega)$ is the PSD matrix function of the multivariate stationary process stochastic. By substituting Equation (19) into Equation (18), after some algebra, the following relationships are obtained:

$$
\begin{aligned}
& \mathrm{E}\left\langle\tilde{\mathbf{X}}_{\mathbf{f}_{\mathrm{o}}}(t) \otimes \tilde{\mathbf{Z}}_{\mathrm{o}}(t)\right\rangle=\int_{-\infty}^{\infty}\left[\mathbf{I}_{n} \otimes \mathbf{H}_{\mathrm{o}}(\omega)\right] \operatorname{Vec}\left\{\mathbf{S}_{\tilde{\mathbf{X}}_{\mathrm{f}_{0}} \tilde{\mathbf{X}}_{\mathrm{f}_{\mathrm{o}}}}(\omega)\right\} \mathrm{d} \omega \\
& \mathrm{E}\left\langle\tilde{\mathbf{Z}}_{\mathrm{o}}(t) \otimes \tilde{\mathbf{X}}_{\mathbf{f}_{\mathrm{o}}}(t)\right\rangle=\int_{-\infty}^{\infty}\left[\mathbf{H}_{\mathrm{o}}^{*}(\omega) \otimes \mathbf{I}_{n}\right] \operatorname{Vec}\left\{\mathbf{S}_{\tilde{\mathbf{X}}_{\mathrm{f}_{0}} \tilde{\mathbf{X}}_{\mathrm{f}_{\mathrm{o}}}}(\omega)\right\} \mathrm{d} \omega,
\end{aligned}
$$

where the asterisk means complex conjugate and

$$
\mathbf{H}_{\mathrm{o}}(\omega)=\left[\left(\mathrm{i} \omega \mathbf{I}_{2 s}-\mathbf{D}_{\mathrm{o}}\right)^{-1} \mathbf{V}_{\mathrm{o}}\right]=\left[\begin{array}{c}
\mathbf{h}_{\mathrm{o}}(\omega) \\
\mathrm{i} \omega \mathbf{h}_{\mathrm{o}}(\omega)
\end{array}\right]
$$

being $\mathbf{h}_{\mathrm{o}}(\omega)$ a matrix of order $s \times n$ defined as

$\mathbf{h}_{\mathrm{o}}(\omega)=\left[\boldsymbol{\Omega}_{\mathrm{o}}^{2}-\omega^{2} \mathbf{I}_{s}+\mathrm{i} \omega \mathbf{E}_{\mathrm{o}}\right]^{-1} \boldsymbol{\Phi}_{\mathrm{o}}^{\mathrm{T}}$

Note that the inverse matrix, which appears in this equation, can be evaluated in closed form for classically damped systems. Indeed, in this case, all the matrices in square brackets are diagonal ones.

Once the statistics vectors, $\mu_{\mathbf{z}_{0}}(t)$ and $\sigma_{\mathbf{z}_{0}}(t)$, in modal subspace are evaluated, the nodal mean value and covariance vectors can be obtained as a

$$
\boldsymbol{\mu}_{\mathbf{y}_{\mathrm{o}}}(t)=\boldsymbol{\Pi}_{\mathrm{o}} \boldsymbol{\mu}_{\mathbf{z}_{\mathrm{o}}}(t) ; \quad \boldsymbol{\sigma}_{\mathbf{y}_{\mathrm{o}}}(t)=\boldsymbol{\Pi}_{\mathrm{o}}^{[2]} \boldsymbol{\sigma}_{\mathbf{z}_{\mathrm{o}}}(t),
$$

where the apex in square brackets means Kronecker power. 


\section{Dynamics modifications}

Let consider now a system obtained changing the geometric and mechanical parameters of the original one. Eventually some structural components can be added or deleted so that the degrees of freedom of the modified system can be different from the original ones. The equation of motion of the modified structural system is written as

$\mathbf{M}_{\mathrm{m}} \ddot{\mathbf{u}}_{\mathrm{m}}(t)+\mathbf{C}_{\mathrm{m}} \dot{\mathbf{u}}_{\mathrm{m}}(t)+\mathbf{K}_{\mathrm{m}} \mathbf{u}_{\mathrm{m}}(t)=\mathbf{f}_{\mathrm{m}}(t)$.

Note that the modifications could lead to a modified non-classically damped system even if the original one is classically damped. Due the structural modifications two main cases can be observed: a) non-topological modifications; b) topological modifications.

\subsection{Non-topological modifications}

In the case of non-topological modification the number of the degrees-offreedom (DOFs) of the modified structural system are the same that one of the original system. Accordingly, the matrices $\mathbf{M}_{\mathrm{m}}, \mathbf{C}_{\mathrm{m}}$ and $\mathbf{K}_{\mathrm{m}}$, of order $n \times n$, and the vector of applied force $\mathbf{f}_{\mathrm{m}}(t)$, of order $n \times 1$, can be written as follows

$\mathbf{M}_{\mathrm{m}}=\mathbf{M}_{\mathrm{o}}+\mathbf{M}_{\mathrm{nn}} ; \quad \mathbf{C}_{\mathrm{m}}=\mathbf{C}_{\mathrm{o}}+\mathbf{C}_{\mathrm{nn}} ; \quad \mathbf{K}_{\mathrm{m}}=\mathbf{K}_{\mathrm{o}}+\mathbf{K}_{\mathrm{nn}} ; \quad \mathbf{f}_{\mathrm{m}}(t)=\mathbf{f}_{\mathrm{o}}(t)+\mathbf{f}_{\mathrm{n}}(t)$,

where the matrices $\mathbf{M}_{\mathrm{nn}}, \mathbf{C}_{\mathrm{nn}}$ and $\mathbf{K}_{\mathrm{nn}}$, of order $n \times n$, account for the variations, induced by the modifications, with respect to the matrices $\mathbf{M}_{\mathrm{o}}, \mathbf{C}_{\mathrm{o}}$ and $\mathbf{K}_{\mathrm{o}}$. It is to emphasize that non-topological modifications are very common in studying MDoF systems with uncertain parameters via a pertinent Monte Carlo simulation. It is well known that for this problem the adoption of Monte Carlo simulation is quite onerous. In this regard, the main steps involved in a Monte Carlo study require: (i) the simulation a set of random variable modelling, (ii) the deterministic analysis of the response for each set of variables, (iii) the evaluation of the response statistics repeating several times deterministic analysis pertinent to each new simulation. In this context the Reanalysis techniques are a promising strategy in reducing the computational effort (Muscolino and Cacciola 2004).

\subsection{Topological modifications}

The modification is called topological if it induces the change of the number of DOFs of the original structural system. This generally happens when structural elements as well as joints are added or deleted. If the number of the DOFs increases the matrices $\mathbf{M}_{\mathrm{m}}, \mathbf{C}_{\mathrm{m}}$ and $\mathbf{K}_{\mathrm{m}}$, of order $g \times g(g>n)$, and the forcing vector $\mathbf{f}_{\mathrm{m}}(t)$, of order $g \times 1$, can be partitioned in the following form 


$$
\begin{array}{ll}
\mathbf{M}_{\mathrm{m}}=\left[\begin{array}{cc}
\mathbf{M}_{\mathrm{o}}+\mathbf{M}_{\mathrm{nn}} & \mathbf{M}_{\mathrm{nr}} \\
\mathbf{M}_{\mathrm{nr}}^{\mathrm{T}} & \mathbf{M}_{\mathrm{rr}}
\end{array}\right] ; & \mathbf{C}_{\mathrm{m}}=\left[\begin{array}{cc}
\mathbf{C}_{\mathrm{o}}+\mathbf{C}_{\mathrm{nn}} & \mathbf{C}_{\mathrm{nr}} \\
\mathbf{C}_{\mathrm{nr}}^{\mathrm{T}} & \mathbf{C}_{\mathrm{rr}}
\end{array}\right] ; \\
\mathbf{K}_{\mathrm{m}}=\left[\begin{array}{cc}
\mathbf{K}_{\mathrm{o}}+\mathbf{K}_{\mathrm{nn}} & \mathbf{K}_{\mathrm{nr}} \\
\mathbf{K}_{\mathrm{nr}}^{\mathrm{T}} & \mathbf{K}_{\mathrm{rr}}
\end{array}\right] ; & \mathbf{f}_{\mathrm{m}}(t)=\left[\begin{array}{c}
\mathbf{f}_{\mathrm{o}}(t)+\mathbf{f}_{\mathrm{n}}(t) \\
\mathbf{f}_{\mathrm{r}}(t)
\end{array}\right] .
\end{array}
$$

where $\mathbf{M}_{\mathrm{nn}}, \mathbf{C}_{\mathrm{nn}}, \mathbf{K}_{\mathrm{nn}}$ and $\mathbf{f}_{\mathrm{n}}(t)$ list the modifications of the structural matrices and the force vector related to the original $n$-DOFs; while $\mathbf{M}_{\mathrm{rr}}, \mathbf{C}_{\mathrm{rr}} \mathbf{K}_{\mathrm{rr}}$, and $\mathbf{f}_{\mathrm{r}}(t)$ are the matrices and the force vector associated with the newly added DOFs; $\mathbf{M}_{\mathrm{nr}}, \mathbf{C}_{\mathrm{nr}}$ and $\mathbf{K}_{\mathrm{nr}}$ represent the coupling between the original and the newly added $r=g-n$ DOFs.

Lastly, in addition to a variation of the structural properties of the original system, some joints can be deleted. It follows that the number of DOFs of the modified structure is lower than that of the original system $(g<n)$, implying a reduction of the problem dimension. The new matrices and the force vector of the modified system are obtained by cancelling out the rows and columns of their original counterparts pertinent to the deleted joints and adding the variations

$$
\mathbf{M}_{\mathrm{m}}=\overline{\mathbf{M}}_{\mathrm{o}}+\overline{\mathbf{M}}_{\mathrm{nn}} ; \quad \mathbf{C}_{\mathrm{m}}=\overline{\mathbf{C}}_{\mathrm{o}}+\overline{\mathbf{C}}_{\mathrm{nn}} ; \quad \mathbf{K}_{\mathrm{m}}=\overline{\mathbf{K}}_{\mathrm{o}}+\overline{\mathbf{K}}_{\mathrm{nn}} ; \mathbf{f}_{\mathrm{m}}(t)=\overline{\mathbf{f}}_{\mathrm{o}}(t)+\overline{\mathbf{f}}_{\mathrm{n}}(t)
$$

In the previous relationships, the overline means that the order of the new matrices is lower than the order of those related to the original structure; the matrices $\overline{\mathbf{M}}_{\mathrm{n}}$, $\overline{\mathbf{C}}_{\mathrm{nn}}$ and $\overline{\mathbf{K}}_{\mathrm{nn}}$ are the matrices of order $g \times g(g<n)$ which denote further possible variations in the modified matrices of the original system.

\subsection{Equations of the modified system}

In this section the equations of motion of the modified system, in modal subspace, with both non-topological and topological modification are formulated in a unified way (Muscolino and Cacciola, 2004; Cacciola et al 2003, 2005). In order to do this, the following coordinate transformation is considered

$$
\mathbf{u}_{\mathrm{m}}(t)=\Psi \mathbf{q}_{\mathrm{m}}(t)
$$

where $\Psi$ is a transformation matrix of order $n \times s$ that satisfy the following condition

$$
\Psi^{T} \mathbf{M}_{\mathrm{m}} \Psi=\mathbf{I}_{s}
$$


Remarkably, for non-topological modifications, the transformation matrix $\Psi$ can be conveniently obtained starting from the matrix of eigenvectors $\Phi_{0}$ as follows

$\Psi=\Phi_{0} \Gamma^{-\mathrm{T}}$

where $\Gamma$ is a triangular matrix of order $n \times n$, evaluated by applying the Cholesky decomposition, that is it satisfies the following condition

$\Gamma \Gamma^{\mathrm{T}}=\boldsymbol{\Phi}_{\mathrm{o}}^{T} \mathbf{M}_{\mathrm{m}} \boldsymbol{\Phi}_{\mathrm{o}}$

In the case in which the system dimension changes, i.e. for topological modifications, then some rows should be cancelled out (if some nodes are deleted) or added (if some nodes are annexed) to the matrix $\Phi_{\mathrm{o}}$ before applying the GramSchmidt procedure. For the case of DOFs addition, the matrix $\Psi$, of order $g \times s$, can be obtained as

$\boldsymbol{\Psi}=\left[\begin{array}{l}\boldsymbol{\Phi}_{\mathrm{o}} \\ \boldsymbol{\Phi}_{\mathrm{r}}\end{array}\right] \boldsymbol{\Gamma}^{-\mathrm{T}}$,

where $\phi_{\mathrm{r}, \mathrm{j}}$, generic column of the new matrix $\boldsymbol{\Phi}_{\mathrm{r}}$ (of order $r \times s$ ), is given by (Chen et al 2000)

$\phi_{\mathrm{r}, j}=-\left(\mathbf{K}_{\mathrm{rr}}-\omega_{\mathrm{o}, j}^{2} \mathbf{M}_{\mathrm{rr}}\right)^{-1}\left(\mathbf{K}_{\mathrm{nr}}^{\mathrm{T}}-\omega_{\mathrm{o}, j}^{2} \mathbf{M}_{\mathrm{nr}}^{\mathrm{T}}\right) \phi_{\mathrm{o}, j} ; \quad(j=1,2, \ldots, s)$,

being $\phi_{0, j}$ and $\omega_{0, j}$ the generic eigenvector and the natural frequency the of the original structural system, respectively. In the case of DOFs reduction, the matrix $\Psi$ is simply obtained deleting the rows of the matrix $\boldsymbol{\Phi}_{\mathrm{o}}$ according to the number of nodes deleted in the modified structures

Once the matrix $\Psi$ is defined, by applying the coordinate transformation (28) the coupled equations of motion for the modified structural system can be rewritten in the reduced modal space as

$$
\ddot{\mathbf{q}}_{\mathrm{m}}(t)+\Psi^{T} \mathbf{C}_{\mathrm{m}} \Psi \dot{\mathbf{q}}_{\mathrm{m}}(t)+\Psi^{T} \mathbf{K}_{\mathrm{m}} \Psi \mathbf{q}_{\mathrm{m}}(t)=\Psi^{T} \mathbf{f}_{\mathrm{m}}(t)
$$

whereas, in the reduced (modal) state space

$$
\dot{\mathbf{z}}_{\mathrm{m}}(t)=\mathbf{D}_{\mathrm{m}} \mathbf{z}_{\mathrm{m}}(t)+\mathbf{V}_{\mathrm{m}} \mathbf{f}_{\mathrm{m}}(t)
$$

being 


$$
\mathbf{D}_{\mathrm{m}}=\left[\begin{array}{cc}
\mathbf{0} & \mathbf{I}_{s} \\
-\boldsymbol{\Psi}^{T} \mathbf{K}_{\mathrm{m}} \boldsymbol{\Psi} & -\boldsymbol{\Psi}^{T} \mathbf{C}_{\mathrm{m}} \boldsymbol{\Psi}
\end{array}\right], \quad \mathbf{V}_{\mathrm{m}}=\left[\begin{array}{c}
\mathbf{0} \\
\boldsymbol{\Psi}^{T}
\end{array}\right]
$$

Note that, in order to numerically evaluate the dynamic response of the modified structural system, the procedure recently proposed by the authors (Muscolino and Cacciola 2004, Cacciola et al 2003,2005) can be applied. This procedure, according to the philosophy of the Reanalysis, requires the following steps: (i) to write the solution in the following form

$$
\mathbf{z}_{\mathrm{m}}(t)=\left[\begin{array}{c}
\mathbf{q}_{\mathrm{m}}(t) \\
\dot{\mathbf{q}}_{\mathrm{m}}(t)
\end{array}\right]=\left[\begin{array}{c}
\mathbf{q}_{\mathrm{o}}(t) \\
\dot{\mathbf{q}}_{\mathrm{o}}(t)
\end{array}\right]+\left[\begin{array}{c}
\Delta \mathbf{q}(t) \\
\Delta \dot{\mathbf{q}}(t)
\end{array}\right]=\mathbf{z}_{\mathrm{o}}(t)+\Delta \mathbf{z}(t),
$$

where $\Delta \mathbf{z}(t)$ is the increment of the response of the modified structural system in the reduced (modal) subspace with respect to the original modal subspace; (ii) to select, for both the original and the modified structural system, the same number of generalized co-ordinates in the reduced subspaces.

\section{Reanalysis of the stochastic stationary response}

The most common structural systems of engineering interest are subjected to stochastic loads. In this context the Stochastic Mechanics deals with the challenging problem determining the random response of a structural system. Several approaches have been proposed in literature to cope with this problem (see e.g. Lin, 1976; Lutes-Sarkani, 1997). The random response is fully defined, by a probabilistic point of view, if the probability density function or alternatively all the statistical moments of the response are known. In the case in which the system is linear possessing deterministic geometry and mechanical parameters, and it is forced by a Gaussian process, the response is Gaussian too. So that, only the statistical moments until the second order are needed to fully characterize the stochastic response. In this section the Reanalysis is formulated for the case of stationary input.

\subsection{First and second order statistical moments for dynamic modifications}

Let assume now that the linear structural system is modified and that the stochastic input is a stationary Gaussian process. According to Equation (16), the first two statistical moments of the modified systems are given by the equations

$$
\begin{gathered}
\boldsymbol{\mu}_{\mathbf{z}_{\mathrm{m}}}=-\mathbf{D}_{\mathrm{m}}^{-1} \mathbf{V}_{\mathrm{m}} \boldsymbol{\mu}_{\mathbf{f}_{\mathrm{m}}}=-\left(\mathbf{D}_{\mathrm{o}}+\Delta \mathbf{D}\right)^{-1} \mathbf{V}_{\mathrm{m}} \boldsymbol{\mu}_{\mathbf{f}_{\mathrm{m}}} \\
\boldsymbol{\sigma}_{\mathbf{z}_{\mathrm{m}}}=-\mathbf{D}_{\mathrm{m}, 2}^{-1} \tilde{\mathbf{F}}_{\mathbf{Z}_{\mathrm{m}}}=-\left(\mathbf{D}_{\mathrm{o}, 2}+\Delta \mathbf{D}_{2}\right)^{-1} \tilde{\mathbf{F}}_{\mathbf{Z}_{\mathrm{m}}}
\end{gathered}
$$


with $\mu_{\mathbf{f}_{\mathrm{m}}}$ the stochastic average of vector $\mathbf{f}_{\mathrm{m}}(t)$ and

$$
\begin{aligned}
& \mathbf{D}_{\mathrm{m}, 2}=\mathbf{I}_{2 s} \otimes \mathbf{D}_{\mathrm{m}}+\mathbf{D}_{\mathrm{m}} \otimes \mathbf{I}_{2 s} ; \\
& \tilde{\mathbf{F}}_{\mathbf{Z}_{\mathrm{m}}}=\left(\mathbf{V}_{\mathrm{m}} \otimes \mathbf{I}_{2 s}\right) \mathrm{E}\left\langle\tilde{\mathbf{X}}_{\mathbf{f}_{\mathrm{m}}}(t) \otimes \tilde{\mathbf{Z}}_{\mathrm{m}}(t)\right\rangle+\left(\mathbf{I}_{2 s} \otimes \mathbf{V}_{\mathrm{m}}\right) \mathrm{E}\left\langle\tilde{\mathbf{Z}}_{\mathrm{m}}(t) \otimes \tilde{\mathbf{X}}_{\mathbf{f}_{\mathrm{m}}}(t)\right\rangle .
\end{aligned}
$$

In the Equations (38) the following positions have been made

$$
\Delta \mathbf{D}=\mathbf{D}_{\mathrm{m}}-\mathbf{D}_{\mathrm{o}} ; \quad \Delta \mathbf{D}_{2}=\mathbf{D}_{\mathrm{m}, 2}-\mathbf{D}_{\mathrm{o}, 2}
$$

The elements of vector $\tilde{\mathbf{F}}_{\mathbf{Z}_{\mathrm{m}}}$, which represents the input-output stochastic averages for the modified system, can be evaluated as

$$
\begin{aligned}
& \mathrm{E}\left\langle\tilde{\mathbf{X}}_{\mathbf{f}_{\mathrm{m}}}(t) \otimes \tilde{\mathbf{Z}}_{\mathrm{m}}(t)\right\rangle=\int_{0}^{t}\left[\mathbf{I}_{n} \otimes\left(\Theta_{\mathrm{m}}(t-\tau) \mathbf{V}_{\mathrm{m}}\right)\right] \mathbf{r}_{\tilde{\mathbf{X}}_{\mathbf{f}_{\mathrm{m}}} \tilde{\mathbf{X}}_{\mathbf{f}_{\mathrm{m}}}(\tau-t) \mathrm{d} \tau ;} \\
& \mathrm{E}\left\langle\tilde{\mathbf{Z}}_{\mathrm{m}}(t) \otimes \tilde{\mathbf{X}}_{\mathbf{f}_{\mathrm{m}}}(t)\right\rangle=\int_{0}^{t}\left[\left(\Theta_{\mathrm{m}}(t-\tau) \mathbf{V}_{\mathrm{m}}\right) \otimes \mathbf{I}_{n}\right] \mathbf{r}_{\tilde{\mathbf{X}}_{\mathbf{f}_{\mathrm{m}}} \tilde{\mathbf{X}}_{\mathbf{f}_{\mathrm{m}}}(t-\tau) \mathrm{d} \tau .}
\end{aligned}
$$

In the latter equation the input-output stochastic average are not time-dependent functions, and can be evaluated as:

$$
\begin{aligned}
& \mathrm{E}\left\langle\tilde{\mathbf{X}}_{\mathbf{f}_{\mathrm{m}}}(t) \otimes \tilde{\mathbf{Z}}_{\mathrm{m}}(t)\right\rangle=\int_{-\infty}^{\infty}\left[\mathbf{I}_{n} \otimes \mathbf{H}_{\mathrm{m}}(\omega)\right] \operatorname{Vec}\left\{\mathbf{S}_{\tilde{\mathbf{x}}_{f_{\mathrm{m}}} \tilde{\mathbf{x}}_{\mathrm{f}_{\mathrm{m}}}}(\omega)\right\} \mathrm{d} \omega ; \\
& \mathrm{E}\left\langle\tilde{\mathbf{Z}}_{\mathrm{m}}(t) \otimes \tilde{\mathbf{X}}_{\mathbf{f}_{\mathrm{m}}}(t)\right\rangle=\int_{-\infty}^{\infty}\left[\mathbf{H}_{\mathrm{m}}^{*}(\omega) \otimes \mathbf{I}_{n}\right] \operatorname{Vec}\left\{\mathbf{S}_{\tilde{\mathbf{X}}_{\mathrm{f}_{\mathrm{m}}} \tilde{\mathbf{x}}_{\mathrm{f}_{\mathrm{m}}}}(\omega)\right\} \mathrm{d} \omega,
\end{aligned}
$$

where

$$
\mathbf{H}_{\mathrm{m}}(\omega)=\left[\left(\mathrm{i} \omega \mathbf{I}_{2 s}-\mathbf{D}_{\mathrm{m}}\right)^{-1} \mathbf{V}_{\mathrm{m}}\right]=\left[\begin{array}{c}
\mathbf{h}_{\mathrm{m}}(\omega) \\
\mathrm{i} \omega \mathbf{h}_{\mathrm{m}}(\omega)
\end{array}\right]
$$

and

$$
\mathbf{h}_{\mathrm{m}}(\omega)=\left[\boldsymbol{\Psi}^{T} \mathbf{K}_{\mathrm{m}} \Psi-\omega^{2} \mathbf{I}_{s}+\mathrm{i} \omega \boldsymbol{\Psi}^{T} \mathbf{C}_{\mathrm{m}} \Psi\right]^{-1} \boldsymbol{\Psi}^{\mathrm{T}}
$$




\subsection{Reanalysis methods}

The objective of the Reanalysis in this context is the evaluation of the statistical moments until the second order of the modified structure taking advantage of the results relative to the original structure, so reducing the computational effort. Being the equations for determining the statistical moments of the response, for stationary input, algebraic ones, the main Reanalysis techniques developed for the deterministic static reanalysis are herein extended to the case of stochastic stationary Gaussian input.

\section{Sherman-Morrison-Woodbury method}

In mathematics, in particular in linear algebra, the Sherman-Morrison-Woodbury (SMW) formula (Sherman and Morrison 1949, 1950; Woodbury 1950) gives the exact inverse of an invertible matrix due to a rank- $r$ change, formulated as the superposition of $r$ change of rank one. It follows that by introducing the vector $\mathbf{v}_{j}$ of order $2 s \times 1$

$$
\mathbf{v}_{j}=\left(\begin{array}{c}
0 \\
0 \\
\vdots \\
1 \\
0
\end{array}\right) j-t h \text { position }
$$

possessing the element in the $j$-th position equal to one and all the other equal to zero, the matrix $\mathbf{D}_{\mathrm{m}}$ can be rewritten in the following form

$$
\mathbf{D}_{\mathrm{m}}=\mathbf{D}_{\mathrm{o}}+\sum_{i=1}^{2 s} \sum_{j=1}^{2 s} \Delta D_{i, j} \mathbf{v}_{i} \mathbf{v}_{j}^{T}
$$

being $\Delta D_{i, j}$ the element of the matrix $\Delta \mathbf{D}$ located at the $i$-th row and $j$-th column. Accordingly to the recursive SMW formula, it is possible to evaluate in exact form the inverse of matrix $\mathbf{D}_{\mathrm{m}}$ updating recursively the inverse of the matrix $\mathbf{D}_{\mathrm{o}}$. That is

$\mathbf{E}_{0}=\mathbf{D}_{\mathrm{o}}^{-1}$;

$$
\mathbf{E}_{k}=\mathbf{E}_{k-1}-\Delta D_{i, j} \frac{\mathbf{E}_{k-1} \mathbf{v}_{i} \mathbf{v}_{j}^{T} \mathbf{E}_{k-1}}{1+\Delta D_{i, j} \mathbf{v}_{j}^{T} \mathbf{E}_{k-1} \mathbf{v}_{i}} ; \quad k=1, \ldots, 4 s^{2}
$$

Where $k$ stands for $k$-th iterations and it is independent of $i$ and $j$. Obviously the procedure should be applied only to the elements $\Delta D_{i, j} \neq 0$. Since most of the 
elements of the matrix $\Delta \mathbf{D}$ are zeros, the number of iterations $k$ are only a few, so saving in the computational effort.

Analogously the covariance vector of the modified system can be obtained updating introducing the vector $\mathbf{v}_{2, j}$ of order $4 s^{2} \times 1$

$$
\mathbf{v}_{2, j}=\left(\begin{array}{c}
0 \\
0 \\
\vdots \\
1 \\
0
\end{array}\right) j-t h \text { position }
$$

possessing the element in the $j$-th position equal to one and all the other equal to zero, the matrix $\mathbf{D}_{\mathrm{m}, 2}$ can be rewritten in the following form

$$
\mathbf{D}_{\mathrm{m}, 2}=\mathbf{D}_{\mathrm{o}, 2}+\sum_{i=1}^{4 s^{2}} \sum_{j=1}^{4 s^{2}} \Delta D_{2, i, j} \mathbf{v}_{2, i} \mathbf{v}_{2, j}^{T}
$$

Being $\Delta D_{2, i, j}$ the element of the matrix $\Delta \mathbf{D}_{2}$ located at the $i$-th row and $j$-th column. By considering, in the double summation (49), only the terms for which $\Delta D_{2, i, j} \neq 0$, the recursive SMW formula leads to

$\mathbf{E}_{2,0}=\mathbf{D}_{0,2}^{-1}$

$$
\mathbf{E}_{2, k}=\mathbf{E}_{2, k-1}-\Delta D_{2, i, j} \frac{\mathbf{E}_{2, k-1} \mathbf{v}_{2, i} \mathbf{v}_{2, j}^{T} \mathbf{E}_{2, k-1}}{1+\Delta D_{2, i, j} \mathbf{v}_{2, j}^{T} \mathbf{E}_{2, k-1} \mathbf{v}_{2, i}} ; \quad k=1, \ldots, 16 s^{4}
$$

So obtaining the exact solution of the second algebraic Equation (38), updating recursively the inverse of the matrix $\mathbf{D}_{\mathrm{o}, 2}$.

\section{Combined Approximations method}

The main idea of the Combined Approximations (CA), proposed by Kirsch (1996) for the static Reanalysis, is to approximate the displacement vector of the modified structure by a linear combination of only few (significantly less than the number of DoFs) linearly independent vectors. The CA provides very accurate results also for structural systems with large changes in the structural system (Kirsh 2008). In order to extend this method to stochastic stationary Reanalysis, the mean value and covariance vectors are herein approximate by two linear combinations of preselected linearly independent basis vectors, generated as 


$$
\begin{aligned}
& \mathbf{r}^{(1)}=-\mathbf{D}_{\mathrm{o}}^{-1} \mathbf{V}_{\mathrm{m}} \boldsymbol{\mu}_{\mathbf{f}_{\mathrm{m}}} ; \quad \mathbf{r}^{(i)}=-\mathbf{D}_{\mathrm{o}}^{-1} \Delta \mathbf{D} \mathbf{r}^{(i-1)} i=2,3, \ldots, p ; \\
& \mathbf{r}_{2}^{(1)}=\mathbf{D}_{\mathrm{o}, 2}^{-1} \tilde{\mathbf{F}}_{\mathbf{Z}_{\mathrm{m}}} ; \quad \mathbf{r}_{2}^{(j)}=-\mathbf{D}_{\mathrm{o}, 2}^{-1} \Delta \mathbf{D}_{2} \mathbf{r}_{2}^{(j-1)} j=2,3, \ldots, p_{2} .
\end{aligned}
$$

Then the solution of algebraic Equations (38), mean value and covariance vectors, can be approximate by these bases of independent vectors as

$\mu_{\mathbf{z}_{\mathrm{m}}} \simeq \mathbf{R} \mathbf{x} ; \quad \sigma_{\mathbf{z}_{\mathrm{m}}} \simeq \mathbf{R}_{2} \mathbf{x}_{2}$

where $\mathbf{R}$ and $\mathbf{R}_{2}$ are two matrices of order $2 s \times p$ and $4 s^{2} \times p_{2}$, respectively defined as

$$
\mathbf{R}=\left[\mathbf{r}^{(1)}, \ldots, \mathbf{r}^{(i)}\right] ; \quad \mathbf{R}_{2}=\left[\mathbf{r}_{2}^{(1)}, \ldots, \mathbf{r}_{2}^{(j)}\right]
$$

The two vectors $\mathbf{x}$ and $\mathbf{x}_{2}$ are of order $p(\ll 2 s)$ and $p_{2}\left(\ll 4 s^{2}\right)$, respectively, that can be evaluated in the reduced subspaces as the solution of two sets of algebraic equations of reduced order with respect the equations governing the problem

$$
\mathbf{x}=-\mathbf{A}^{-1} \mathbf{R}^{\mathrm{T}} \mathbf{V}_{\mathrm{m}} \boldsymbol{\mu}_{\mathrm{f}_{\mathrm{m}}} ; \quad \mathbf{x}_{2}=-\mathbf{A}_{2}^{-1} \mathbf{R}_{2}^{\mathrm{T}} \tilde{\mathbf{F}}_{\mathbf{Z}_{\mathrm{m}}} .
$$

In these equations, $\mathbf{A}$ and $\mathbf{A}_{2}$ are two matrices of order $p \times p$ and $p_{2} \times p_{2}$, respectively, given as

$$
\mathbf{A}=\mathbf{R}^{\mathrm{T}} \mathbf{D}_{\mathrm{m}} \mathbf{R} ; \quad \mathbf{A}_{2}=\mathbf{R}_{2}^{\mathrm{T}} \mathbf{D}_{\mathrm{m}, 2} \mathbf{R}_{2} .
$$

\section{Numerical results}

The proposed procedure is herein applied to the bridge-like truss structure depicted in Figure 1. The structure is assumed proportionally damped with damping ratio $\zeta_{0}=0.02$ set equal for the first two modes. The structure undergoes to base nonuniform base excitation modelled by a zero-mean, tri-variate Gaussian stationary process defined by the following cross-spectral density matrix (see e.g. Deodatis 1996): 
$\mathbf{S}_{i_{g}}(\omega)=\left[\begin{array}{lll}S_{11}(\omega) & S_{12}(\omega) & S_{13}(\omega) \\ S_{21}(\omega) & S_{22}(\omega) & S_{23}(\omega) \\ S_{31}(\omega) & S_{32}(\omega) & S_{33}(\omega)\end{array}\right]$

The Clough and Penzien (1975) acceleration spectrum is selected to model the power spectral density functions $S_{j j}(\omega),(j=1,2,3)$ :

$$
S_{j j}(\omega) \equiv S_{j}(\omega)=S_{0 j} \frac{\left(1+4 \zeta_{g j}^{2}\left(\frac{\omega}{\omega_{g j}}\right)^{2}\right)}{\left(1-\left(\frac{\omega}{\omega_{g j}}\right)^{2}\right)^{2}+4 \zeta_{g j}^{2}\left(\frac{\omega}{\omega_{g j}}\right)^{2}} \frac{\left(\frac{\omega}{\omega_{f j}}\right)^{4}}{\left(1-\left(\frac{\omega}{\omega_{f j}}\right)^{2}\right)^{2}+4 \zeta_{f j}^{2}\left(\frac{\omega}{\omega_{f j}}\right)^{2}}
$$

In which the following filter parameters have been assumed

$$
\begin{aligned}
& \omega_{g, 1}=8 \pi \mathrm{rad} / \mathrm{s}, \omega_{g, 2}=5 \pi \mathrm{rad} / \mathrm{s}, \omega_{g, 3}=2.4 \pi \mathrm{rad} / \mathrm{s} \\
& \zeta_{g, 1}=\zeta_{g, 2}=0.6, \zeta_{g, 3}=0.85 \\
& \omega_{f, i}=0,1 \omega_{g, i} ; \quad \zeta_{f, i}=\zeta_{g, i} ; \quad(i=1,2,3)
\end{aligned}
$$

Moreover,

$S_{01}=0.00623 \mathrm{~cm}^{2} / \mathrm{s}^{3}, S_{02}=0.00997 \mathrm{~cm}^{2} / \mathrm{s}^{3}, S_{03}=0.01845 \mathrm{~cm}^{2} / \mathrm{s}^{3}$

represent three different soil profiles: i.e. rock or stiff soil conditions (1), deep cohesionless soils (2) and soft to medium clays and sands (3) as evidenced in Figure 2 .

The off-diagonal terms are defined as

$S_{j k}(\omega) \equiv S_{k j}(\omega)=S_{j j}(\omega) \gamma_{j k}(\omega)$

where $\gamma_{j k}(\omega)$ is the coherence function. The Harichandran andVanmarcke (1986) model is chosen to model the coherence function:

$$
\gamma_{j k}(\omega)=a \exp \left[-\frac{2 \xi_{j k}}{\alpha \theta(\omega)}(1-a+\alpha a)\right]+(1-a) \exp \left[-\frac{2 \xi_{j k}}{\theta(\omega)}(1-a+\alpha a)\right]
$$


where $\xi_{j k}$ being the distance between points $j$ and $k$ (here it is assumed: $\xi_{12}=\xi_{23}=15 m$ and $\left.\xi_{13}=30 m\right)$ and $\theta(\omega)$ is given by the following equation

$\theta(\omega)=k\left[1+\left(\frac{\omega}{\omega_{0}}\right)^{b}\right]^{-1 / 2}$.

Furthermore, the following parameters have been assumed

$a=0.626 ; \quad \alpha=0.022 ; \quad k=19700 \mathrm{~m} ; \omega_{0}=12.692 \mathrm{rad} / \mathrm{s}, \quad b=3.47$

In Figure 3 the coherence functions are plotted. Note that the lack of coherency of the ground motion accelerations at the three supports is mainly due to the different soil profile underneath the supports of the truss.

After defined the seismic action the second order statistical moments have been determined through the procedure described in section 2.2. Specifically the equations governing the motion of the original structure read

$\mathbf{M}_{\mathrm{o}} \ddot{\mathbf{u}}_{\mathrm{o}}(t)+\mathbf{C}_{\mathrm{o}} \dot{\mathbf{u}}_{\mathrm{o}}(t)+\mathbf{K}_{\mathrm{o}} \mathbf{u}_{\mathrm{o}}(t)=-\mathbf{M}_{\mathrm{o}} \mathbf{T}_{\mathrm{o}} \ddot{\mathbf{u}}_{g}(t) ; \quad \mathbf{u}_{\mathrm{o}}(0)=\mathbf{0} ; \dot{\mathbf{u}}_{\mathrm{o}}(0)=\mathbf{0}$,

Where $\ddot{\mathbf{u}}_{g}(t)$ is the vector of order $3 \times 1$ listing the ground motion accelerations at the supports, while $\mathbf{T}_{\mathrm{o}}$ is given by the following relationship

$\mathbf{T}_{\mathrm{o}}=-\mathbf{K}_{\mathrm{o}}^{-1} \mathbf{k}_{g, \mathrm{o}}$

in which $\mathbf{k}_{g, 0}$ is the matrix of order $n \times 3$ taking into account of the forces induced in the structures due a unitary displacement of each individual support while the other are imposed to be zero. Therefore classical modal analysis retaining the first 10 modes is applied and the analysis is conducted in the reduced modal state space. Accordingly the vector $\mathbf{V}_{\mathrm{o}}$ and the stochastic averages $\mathrm{E}\left\langle\tilde{\mathbf{X}}_{\mathbf{f}_{\mathrm{o}}}(t) \otimes \tilde{\mathbf{Z}}_{\mathrm{o}}(t)\right\rangle$ and $\mathrm{E}\left\langle\tilde{\mathbf{Z}}_{\mathrm{o}}(t) \otimes \tilde{\mathbf{X}}_{\mathbf{f}_{\mathrm{o}}}(t)\right\rangle$ are modified as follows, respectively

$$
\mathbf{V}_{\mathrm{o}}=\left[\begin{array}{c}
\mathbf{0} \\
-\boldsymbol{\Phi}_{\mathrm{o}}^{\mathrm{T}} \mathbf{M}_{\mathrm{o}} \mathbf{T}_{\mathrm{o}}
\end{array}\right]
$$

and

$$
\begin{aligned}
& \mathrm{E}\left\langle\tilde{\mathbf{X}}_{\dot{\mathbf{u}}_{\mathrm{g}}}(t) \otimes \tilde{\mathbf{Z}}_{\mathrm{o}}(t)\right\rangle=\int_{-\infty}^{\infty}\left[\mathbf{I}_{3} \otimes \mathbf{H}_{\mathrm{o}}(\omega)\right] \operatorname{Vec}\left\{\mathbf{S}_{\tilde{\mathbf{X}}_{\mathbf{u}_{\mathrm{g}}} \tilde{\mathbf{x}}_{\mathbf{u}_{\mathrm{g}}}}(\omega)\right\} \mathrm{d} \omega ; \\
& \mathrm{E}\left\langle\tilde{\mathbf{Z}}_{\mathrm{o}}(t) \otimes \tilde{\mathbf{X}}_{\mathbf{u}_{\mathrm{i}}}(t)\right\rangle=\int_{-\infty}^{\infty}\left[\mathbf{H}_{\mathrm{o}}^{*}(\omega) \otimes \mathbf{I}_{3}\right] \operatorname{Vec}\left\{\mathbf{S}_{\tilde{\mathbf{X}}_{\tilde{\mathbf{u}}_{\mathrm{g}}} \tilde{\mathbf{x}}_{\mathbf{u}_{\mathrm{g}}}}(\omega)\right\} \mathrm{d} \omega,
\end{aligned}
$$


The nodal mean value and covariance vectors have been then determined applying Equations(23).

In Figure 4 are shown three plausible modifications that can be applied to the original structures. In all the case the modified structure is assumed classically damped. Thus any variation in the stiffness and/or mass matrix induces a variation in the damping matrix as well. In the first case (Figure 4a) a change in the geometry is imposed. Specifically all the upper joints are translated of one meter. As a consequence the mass, stiffness and damping matrices are modified. In this case the number of the degrees of freedom does not change so to belonging to the class of non-topological modifications. In the second and third case (Figures 4b,c) topological modifications are introduced. In the second case (Figures 4b) some elements and joints have been deleted so reducing the number of the degrees of freedom, while in the last case (Figure 4c) some elements and joints have been added so to increase the number of the degrees of freedom. Then the overall scenario of possible modifications has been considered in this illustrative example. Second order statistics of the modified structural systems have been the determined through the proposed reanalysis techniques and compared with the exact solution determined through the procedure described in section 2.2 replacing the indexes "o" with " $\mathrm{m}$ " by considering 10 modes. Since the multi-correlated is zero mean process only the response covariance vectors have to be evaluated. In Table (1-3) are compared the variance of the 5-th and 13-th joint of the truss structures of Figure 4 with the "exact" ones. Remarkably both the procedure provided result in perfect agreement with the exact ones. Interestingly, the CA has been applied by using just 3 base vectors (i.e. $p_{2}=3$ ) so drastically reducing the computational effort.

\section{Conclusions}

The main purpose of the Reanalysis is determining the structural response of modified systems using the pertinent results from the original structure, so reducing the computational effort.

In this paper a procedure for determining the stationary first and second order response statistical moment of linear behaving modified systems under multicorrelated stationary Gaussian processes is proposed. Preliminarily it has been shown that by applying extensively the Kroneker algebra the response statistics vectors can be evaluated by solving two sets of algebraic equations. The proposed procedure requires the following main steps: (i) projection of the equations governing the evolution of the statistical moments in a reduced space, for both original and modified structure, by means of a coordinate transformation which require the evaluation of the eigenproperties of original structure only; (ii) being the equations governing the statistics of the response algebraic ones, the main methods of static Reanalysis (Sherman-Morrison-Woodbury formula and combined approximation method) are reformulated in order to evaluate the statistics of the modified structural system. 
A bridge-like truss structure under non-uniform earthquake excitation has been used for testing the proposed procedure. The numerical results have shown that the accuracy archived is very high for both methods. Moreover, the computational effort can be drastically reduced, without loss in accuracy, considering only few terms of the combined approximation method.

\section{References}

Akgun MA, Garcelon JH, Haftka RT (2001). Fast exact linear and non-linear structural reanalysis and the Sherman-Morrison-Woodbury formulas. International Journal Numerical Methods Engineering 5:1587-1606.

Brewer JW (1978). Kronecker Products and Matrix Calculus in System Theory. IEEE Transactions on Circuits and Systems 9: 772-781.

Cacciola P, Impollonia N, Muscolino G, "A reanalysis technique for structures under with noise excitation", Second M.I.T. Conference on Computational Fluid and Solid Mechanics, Cambridge, Massachussetts, USA 17-20 giugno 2003

Cacciola P, Impollonia N, Muscolino G (2005). A dynamic reanalysis technique for general structural modifications under deterministic and stochastic input. Computers and Structures 83:1076-1085.

Clough RW and Penzien J, (1975), Dynamics of structures, McGraw-Hill.Inc, New York, NY.

Deodatis G (1996), Non-stationary stochastic vector processes: seismic ground motion applications", Probabilistic Engineering Mechanics, 11: 149-168

Chen SH, Huang C, Cao Z (2000). Structural modal reanalysis of topological modifications, Shock and Vibration 7:15-21.

Harichandran RS, Vanmarcke EH (1986), Stochastic variation of earthquake ground motion in space and time. J. Engng Mech., ASCE, 112: 154-74.

Kirsch U (1996). Effective Reanalysis of Structures; Concepts and Implementation. Department of Civil Engineering, Technion, Haifa, Israel.

Kirsh U (2008). Reanalysis of Structures-A unified approach for linear, nonlinear, static and dynamic systems. Springer, Dordrecht.

Kołakowski P, Wikło M, Holnicki-Szulc (2008). The virtual distortion method—a versatile reanalysis tool for structures and systems. Structural Multidisciplinary Optimization 36:217-234, DOI 10.1007/s00158-007-0158-7

Lin YK (1976). Probabilistic theory of structural dynamics., Huntington, UK: Krieger Publisher.

Lutes LD, Sarkani S (1997). Stochastic analysis of structural and mechanical vibrations, Prentice-Hall, Upper Sudale River.

Muscolino G (1996). Dynamically Modified Linear Structures: Deterministic and Stochastic Response. Journal of Engineering Mechanics (ASCE) 122:1044-1051.

Muscolino G (2001).Stochastic Dynamics for Structural Engineering Problems: A Review. Chapter 12 in Civil and Structural Engineering Computing 2001 (edited by B.H.V. Topping), Stirling, Scotland, 2001, Saxe-Coburg Publications: 287-318.

Muscolino G, Cacciola P (2004). Re-Analysis Techniques in Structural Dynamics, Chapter 2 in Progress in Computational Structures Technology (edited by B.H.V. Topping and C.A. Mota Soares), Stirling-Scotland, Saxe-Coburg Publications: 31-58.

Sherman J, Morrison WJ (1949). Adjustment of an inverse matrix corresponding to changes in the elements of a given column or a given row of the original matrix. The Annals of Mathematical Statistics 20:621. 
Sherman J, Morrison WJ (1950). Adjustment of an inverse matrix corresponding to a change in one element of a given matrix. The Annals of Mathematical Statistics 21:124127.

Woodbury M (1950). Inverting modified matrices. Memorandum report 42. Statistical Research Group, Princeton University, NJ, USA 


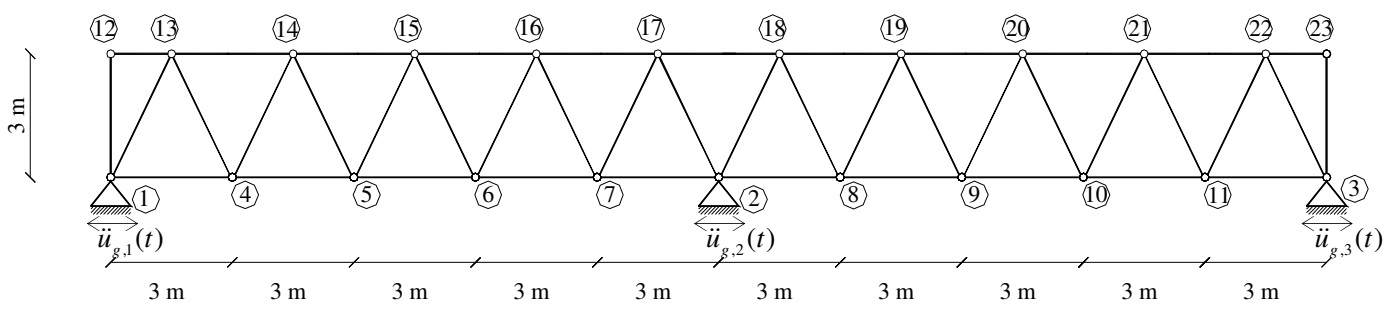

Figure 1: Reference truss structure: cross section area of each member $A=0.004265 \mathrm{~m}^{2}$ and Young modulus $E=2 \times 10^{11} \mathrm{~N} / \mathrm{m}^{2}$ 


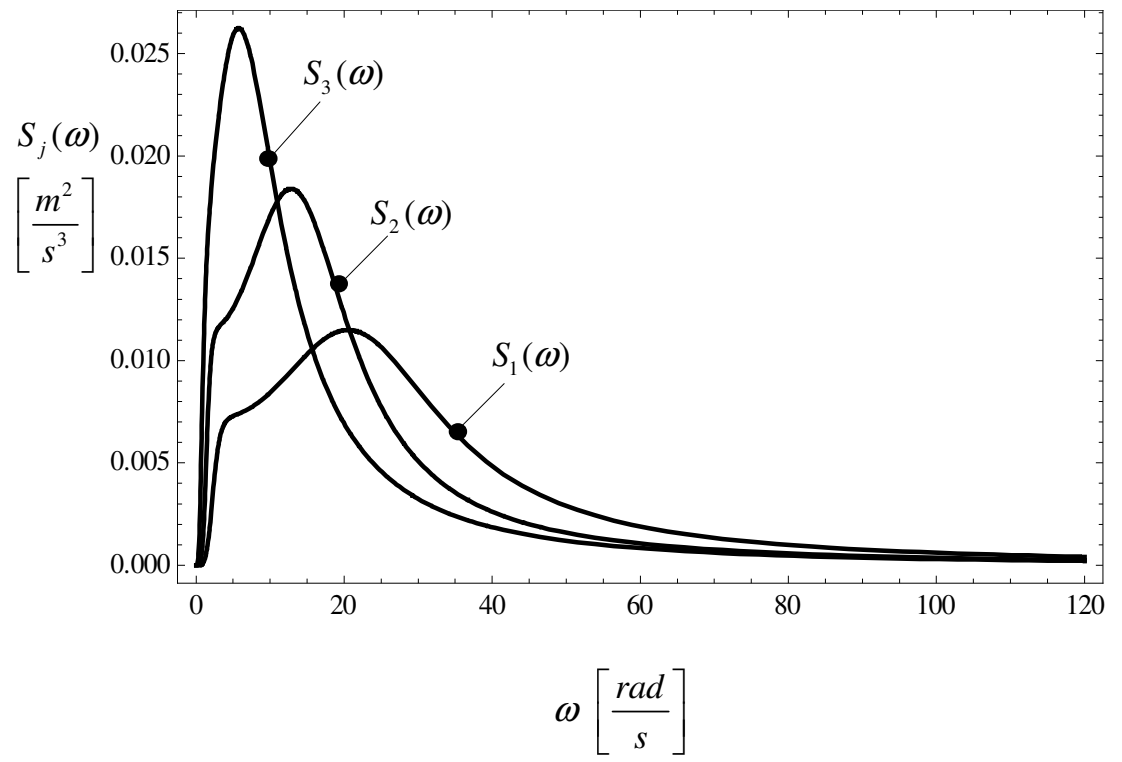

Figure 2: Power spectral densities at the tree supports: (1) rock or stiff soil conditions, (2) deep cohesionless soils, (3) soft to medium clays and sands. 


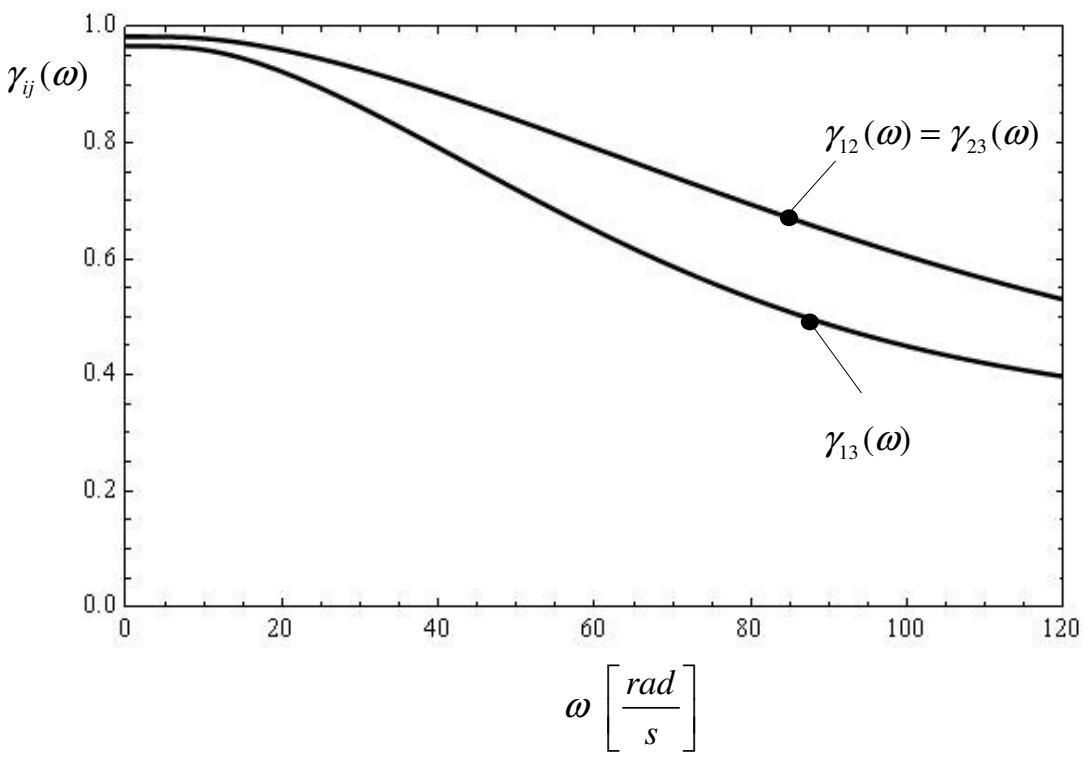

Figure 3: Coherence functions 


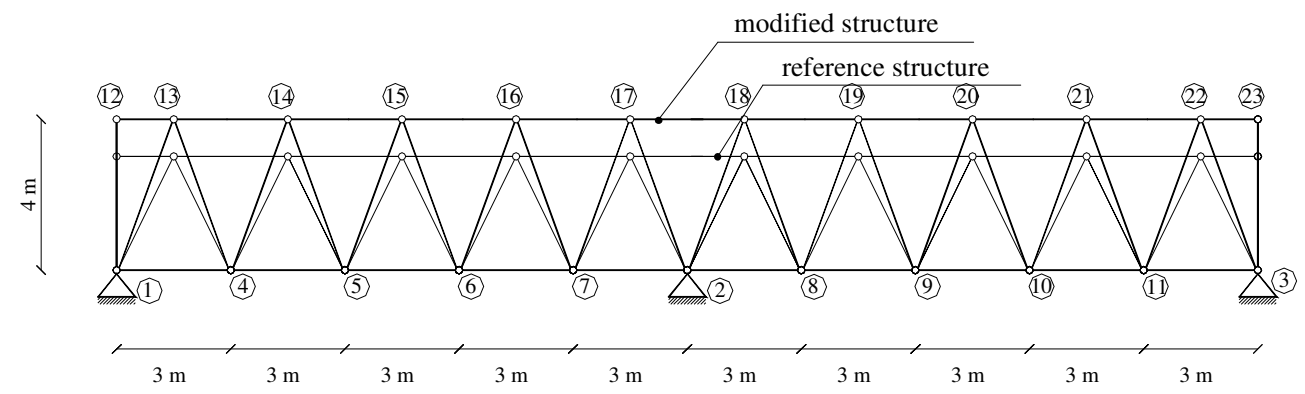

a)

deleted elements and joint
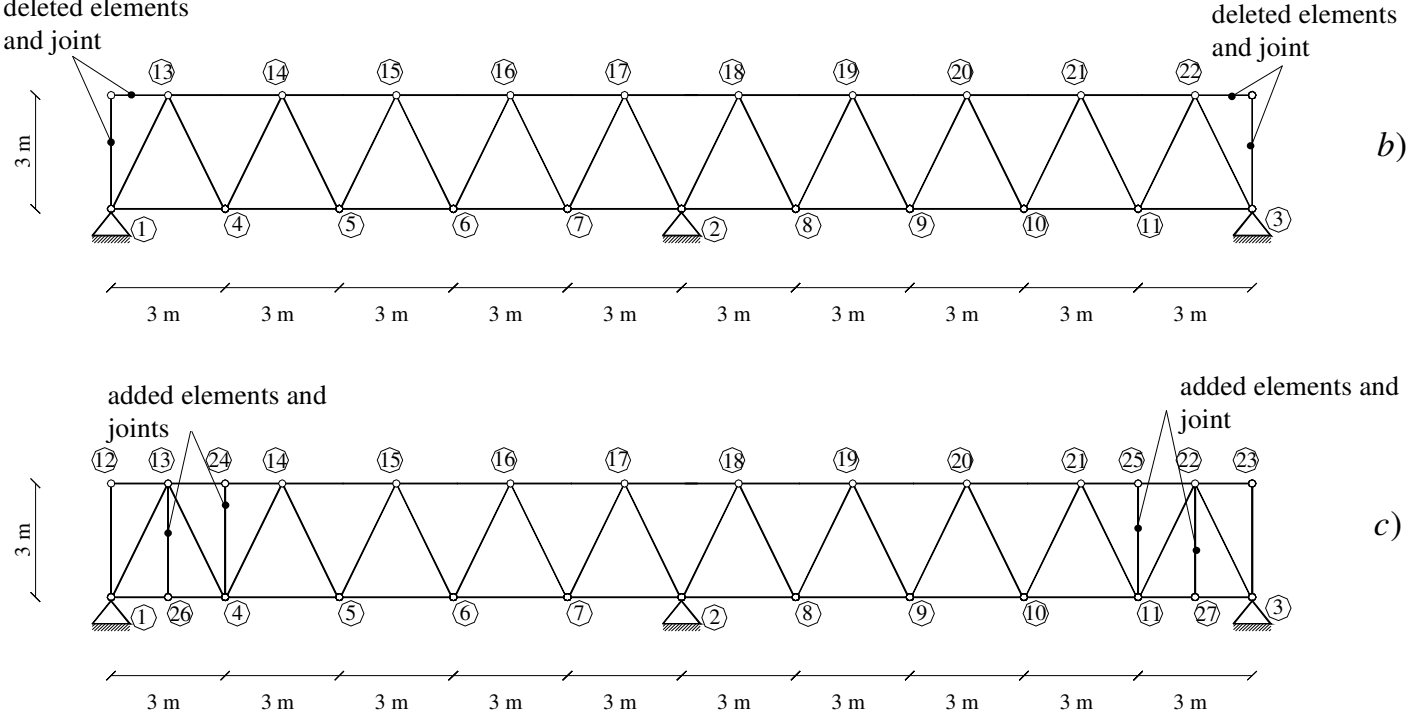

Figure 4: Modified truss structures: a) Non topological modification; b) Topological modification reducing the degrees of freedom; c) Topological modification increasing the degrees of freedom. 
Table 1: Comparison of selected second order moment from exact and approximated reanalysis: non topological modifications.

\begin{tabular}{|c|c|c|c|}
\hline $\begin{array}{c}\text { Second order } \\
\text { moments }\left[\mathrm{m}^{2}\right]\end{array}$ & exact & reanalysis $(C A)$ & reanalysis $(S M W)$ \\
\hline$E\left[u_{5, x}^{2}\right]$ & $2.55344 \times 10^{-8}$ & $2.4953 \times 10^{-8}$ & $2.49945 \times 10^{-8}$ \\
$E\left[u_{5, y}^{2}\right]$ & $8.8094 \times 10^{-8}$ & $8.62552 \times 10^{-8}$ & $8.56168 \times 10^{-8}$ \\
$E\left[u_{13, x}^{2}\right]$ & $1.18824 \times 10^{-8}$ & $1.16652 \times 10^{-8}$ & $1.16623 \times 10^{-8}$ \\
$E\left[u_{13, y}^{2}\right]$ & $6.44497 \times 10^{-8}$ & $6.41159 \times 10^{-8}$ & $6.3719 \times 10^{-8}$ \\
\hline
\end{tabular}

Table 2: Comparison of selected second order moment from exact and approximated reanalysis: topological modifications reducing the degrees of freedom.

\begin{tabular}{|c|c|c|c|}
\hline $\begin{array}{c}\text { Second order } \\
\text { moments }\left[\mathrm{m}^{2}\right]\end{array}$ & exact & reanalysis $(C A)$ & reanalysis $($ SMW $)$ \\
\hline$E\left[u_{5, x}^{2}\right]$ & $2.46931 \times 10^{-8}$ & $2.46851 \times 10^{-8}$ & $2.46851 \times 10^{-8}$ \\
$E\left[u_{5, y}^{2}\right]$ & $1.68161 \times 10^{-7}$ & $1.68159 \times 10^{-7}$ & $1.68159 \times 10^{-7}$ \\
$E\left[u_{13, x}^{2}\right]$ & $1.27049 \times 10^{-8}$ & $1.27173 \times 10^{-8}$ & $1.27173 \times 10^{-8}$ \\
$E\left[u_{13, y}^{2}\right]$ & $1.16532 \times 10^{-7}$ & $1.16531 \times 10^{-7}$ & $1.16531 \times 10^{-7}$ \\
\hline
\end{tabular}

Table 3: Comparison of selected second order moment from exact and approximated reanalysis: topological modifications increasing the degrees of freedom.

\begin{tabular}{|c|c|c|c|}
\hline $\begin{array}{c}\text { Second order } \\
\text { moments }\left[\mathrm{m}^{2}\right]\end{array}$ & exact & reanalysis $(C A)$ & reanalysis $($ SMW $)$ \\
\hline$E\left[u_{5, x}^{2}\right]$ & $2.50065 \times 10^{-8}$ & $2.50222 \times 10^{-8}$ & $2.50222 \times 10^{-8}$ \\
$E\left[u_{5, y}^{2}\right]$ & $1.68155 \times 10^{-7}$ & $1.68157 \times 10^{-7}$ & $1.68157 \times 10^{-7}$ \\
$E\left[u_{13, x}^{2}\right]$ & $1.28498 \times 10^{-8}$ & $1.28375 \times 10^{-8}$ & $1.28375 \times 10^{-8}$ \\
$E\left[u_{13, y}^{2}\right]$ & $1.16614 \times 10^{-7}$ & $1.16613 \times 10^{-7}$ & $1.16613 \times 10^{-7}$ \\
\hline
\end{tabular}


